\title{
Electrical neutrality and pion modes in the two flavor PNJL model
}

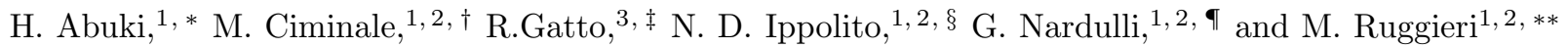 \\ ${ }^{1}$ I.N.F.N., Sezione di Bari, I-70126 Bari, Italia \\ ${ }^{2}$ Università di Bari, I-70126 Bari, Italia \\ ${ }^{3}$ Département de Physique Théorique, Université de Genève, CH-1211 Genève 4, Suisse
}

\begin{abstract}
We study the phase diagram and the pion modes in the electrically neutral two flavor PNJL model. One of the main result of this paper is that in the model with massive quarks, when electrical neutrality is required, pions do not condense in the ground state of the model: the isospin chemical potential $\mu_{I}=-\mu_{e} / 2$ is always smaller than the value required for pion condensation to occur. Moreover we investigate on the pions and $\sigma$ mass spectra. We find that the qualitative behavior of the masses resembles that obtained in the NJL model. We close this paper by studying the intriguing possibility that bound states with the quantum numbers of the pions can be formed above the chiral phase transition.
\end{abstract}

\section{INTRODUCTION}

The use of effective models to understand the phases of Quantum Chromodynamics (QCD) is nowadays a popular tool. Among them, the Nambu-Jona Lasinio model (NJL) is widely used since it allows for a simple discussion of chiral symmetry breaking [1, 2]. The main defect of the NJL model is that it completely ignores glue dynamics: gluons are not introduced in the lagrangian, and the QCD quark-quark interaction mediated by gluons is replaced by an effective four fermion interaction.

An improvement of the NJL model is provided by the introduction of a background temporal static gluon field, coupled to quarks via the QCD covariant derivative. The gluon field is related to the expectation value of Polyakov loop $\Phi[\underline{3}$, and the new model is called Polyakov-NJL (PNJL in the following) [4, [5, 6, 6, 8, 8, 9, 10, 11, 12, 13, 14, 15, 16, 17] It is well known that the Polyakov loop serves as an order parameter for confinement $\rightarrow$ deconfinement transition in the pure glue theory [3]. In more detail, the confined phase is characterized by $\langle\Phi\rangle=0$ and a $Z_{3}$ symmetry; on the other hand in the deconfined phase $\langle\Phi\rangle \neq 0$ and the symmetry $Z_{3}$ is broken. In presence of dynamical quarks, $Z_{3}$ symmetry is explicitly broken and $\Phi$ can not be used as an order parameter. Nevertheless it is commonly used as an indicator of the deconfinement transition.

In the PNJL model an effective potential for $\Phi$ is added by hand to the quark lagrangian, and $\Phi$ is coupled to the quarks via the QCD covariant derivative. The value of $\langle\Phi\rangle$ in the ground state, as well as other quantities of interest like the constituent quark masses, are obtained by minimization of the thermodynamic potential, or equivalently by solving coupled gap equations as in the NJL case. Despite the apparent complication due to the increase of the degrees of freedom, the PNJL model offers a better description of QCD then NJL does since it allows to derive, in the framework of field theory, several results obtained in lattice simulations [6, 7, 8, 9, 11, 12, 14].

The knowledge of the phase diagram of the PNJL model as well as of its bulk properties is of a certain interest. In this paper we study the phase diagram and the pion modes in the electrically neutral two flavor PNJL model. The motivation of our study is straightforward: we are interested to investigate the equilibrium ground state of the model, and in the ground state a net electric charge can not be present, whichever the value of the temperature and of the quark chemical potential is. We consider the possibility of chiral as well as pion condensation in the ground state, at zero and non zero quark chemical potential. A related study without electrical neutrality and at zero chemical potential has been done in Ref. [14]; see also Ref. [18] for a similar study in the framework of the electrically neutral NJL model in the chiral limit.

One of the results of our work is that when electrical neutrality is required, pion do not condense in the ground state of the model: the isospin chemical potential $\mu_{I}=-\mu_{e} / 2$, with $\mu_{e}$ the electron chemical potential, is always smaller than the value required for pion condensation to occur. Moreover we investigate on the pions and $\sigma$ mass

\footnotetext{
*Electronic address: hiroaki.abuki@ba.infn.it

†Electronic address: marco.ciminale@ba.infn.it

$\ddagger$ Electronic address: raoul.gatto@physics.unige.ch

$\S$ Electronic address: nicola.ippolito@ba.infn.it

ๆ Electronic address: giuseppe.nardulli@ba.infn.it

**Electronic address: marco.ruggieri@ba.infn.it
} 
spectra. We find that the qualitative behavior of the masses resembles that obtained in the NJL model. We close this paper by studying the possibility that a bound state with the quantum numbers of the pions can be formed above the chiral phase transition.

The plan of the paper is as follows: in Section [I] we briefly describe the PNJL model; in Section III we show the phase diagram of the model, and some results for quantities of interest (in medium quark mass, pion condensate, mean value of the Polyakov loop, electron chemical potential). In Section[V]we compute the masses of the pseudo-Goldstone modes of the model and of the $\sigma$ mode. Finally in Section $\mathrm{V}$ we draw our conclusions.

\section{FORMALISM}

The Lagrangian density of the two flavor PNJL model is given by [5]

$$
\mathcal{L}^{\prime}=\bar{e}\left(i \gamma_{\mu} \partial^{\mu}\right) e+\bar{\psi}\left(i \gamma_{\mu} D^{\mu}+\mu \gamma_{0}-m\right) \psi+G\left[(\bar{\psi} \psi)^{2}+\left(\bar{\psi} i \gamma_{5} \vec{\tau} \psi\right)^{2}\right]-\mathcal{U}[\Phi, \bar{\Phi}, T]
$$

In the above equation $e$ denotes the electron field; $\psi$ is the quark spinor with Dirac, color and flavor indices (implicitly summed). $m$ corresponds to the bare quark mass matrix; we assume from the very beginning $m_{u}=m_{d}$. The covariant derivative is defined as usual as $D_{\mu}=\partial_{\mu}-i A_{\mu}$. The gluon background field $A_{\mu}=\delta_{0 \mu} A_{0}$ is supposed to be homogeneous and static, with $A_{0}=g A_{0}^{a} T_{a}$ and $T_{a}, a=1, \ldots, 8$ being the $S U(3)$ color generators with the normalization condition $\operatorname{Tr}\left[T_{a} T_{b}\right]=\delta_{a b} . \vec{\tau}$ is a vector of Pauli matrices in flavor space. Finally $\mu$ is the chemical mean quark chemical potential, related to the conserved baryon number.

In Eq. (11) $\Phi, \bar{\Phi}$ correspond to the normalized traced Polyakov loop $L$ and its hermitian conjugate respectively, $\Phi=\operatorname{Tr} L / N_{c}, \bar{\Phi}=\operatorname{Tr} L^{\dagger} / N_{c}$, with

$$
L=\mathcal{P} \exp \left(i \int_{0}^{\beta} A_{4} d \tau\right)=\exp \left(i \beta A_{4}\right), \quad A_{4}=i A_{0},
$$

and $\beta=1 / T$. The term $\mathcal{U}[\Phi, \bar{\Phi}, T]$ is the effective potential for the traced Polyakov loop; in absence of dynamical quarks it is built in order to reproduce the pure glue lattice data of QCD, namely thermodynamical quantities (pressure, entropy and energy density) and the deconfinement temperature of heavy (non-dynamical) quarks, $T=270$ $\mathrm{MeV}$. Several forms of this potential have been suggested in the literature, see for example [5, 6, 7, 8]. In this paper we adopt the following logarithmic form [7],

$$
\frac{\mathcal{U}[\Phi, \bar{\Phi}, T]}{T^{4}}=-\frac{b_{2}(T)}{2} \bar{\Phi} \Phi+b(T) \log \left[1-6 \bar{\Phi} \Phi+4\left(\bar{\Phi}^{3}+\Phi^{3}\right)-3(\bar{\Phi} \Phi)^{2}\right]
$$

with

$$
b_{2}(T)=a_{0}+a_{1}\left(\frac{\bar{T}_{0}}{T}\right)+a_{2}\left(\frac{\bar{T}_{0}}{T}\right)^{2}, \quad b(T)=b_{3}\left(\frac{\bar{T}_{0}}{T}\right)^{3} .
$$

Numerical values of the coefficients are as follows [7]:

$$
a_{0}=3.51, \quad a_{1}=-2.47, \quad a_{2}=15.2, \quad b_{3}=-1.75
$$

If dynamical quarks were not present then one should chose $\bar{T}_{0}=270 \mathrm{MeV}$ in order to reproduce the deconfinement transition at $T=270 \mathrm{MeV}$. In presence of quarks $\bar{T}_{0}$ gets a dependence on the number of active flavors, as shown in Refs. [6, 10]. Following Ref. [10] we chose $\bar{T}_{0}=208 \mathrm{MeV}$ in Eq. (4), which is appropriate to deal with two degenerate flavors.

As explained in the Introduction we are interested to study the ground state of the model specified by the Lagrangian in Eq. (11), at each value of the temperature $T$ and the chemical potential $\mu$, corresponding to a vanishing total electric charge. To this end we introduce electrons in Eq. (11) since a net number of electrons could be needed, beside the pion condensate (if any, see below), to ensure the electrical neutrality of the ground state. In order to build the neutral ground state we work as usual in the gran canonical ensemble formalism, adding to Eq. (11) the term $\mu_{Q} N_{Q}$, $\mu_{Q}$ being the chemical potential for the total charge $N_{Q}$, and requiring stationarity of the thermodynamic potential with respect to variations of $\mu_{Q}$, which is equivalent to the requirement $\left\langle N_{Q}\right\rangle=0$ in the ground state. To be more specific, since the total charge operator is given in terms of the quark and electron fields by

$$
N_{Q}=\frac{2}{3} N_{u}-\frac{1}{3} N_{d}-N_{e}=\frac{2}{3} u^{\dagger} u-\frac{1}{3} d^{\dagger} d-e^{\dagger} e,
$$


it is easy to recognize that the lagrangian $\mathcal{L}$ in the gran canonical ensemble $\mathcal{L}=\mathcal{L}^{\prime}+\mu_{Q} N_{Q}$ can be written as

$$
\mathcal{L}=\bar{e}\left(i \gamma_{\mu} \partial^{\mu}+\mu_{e} \gamma_{0}\right) e+\bar{\psi}\left(i \gamma_{\mu} D^{\mu}+\hat{\mu} \gamma_{0}-m\right) \psi+G\left[(\bar{\psi} \psi)^{2}+\left(\bar{\psi} i \gamma_{5} \vec{\tau} \psi\right)^{2}\right]-\mathcal{U}[\Phi, \bar{\Phi}, T]
$$

where $\mu_{e}=-\mu_{Q}$ and the quark chemical potential matrix $\hat{\mu}$ is defined in flavor-color space as

$$
\hat{\mu}=\left(\begin{array}{cc}
\mu-\frac{2}{3} \mu_{e} & 0 \\
0 & \mu+\frac{1}{3} \mu_{e}
\end{array}\right) \otimes \mathbf{1}_{c}
$$

where $\mathbf{1}_{c}$ denotes identity matrix in color space.

In this paper we work in the mean field approximation. In order to study chiral symmetry breaking and to allow for pion condensation we assume that in the ground state the expectation values for the following operators may develop [14, 18],

$$
\sigma=\langle\bar{\psi} \psi\rangle, \quad \pi=\left\langle\bar{\psi} i \gamma_{5} \tau_{1} \psi\right\rangle
$$

In the above equation a summation over flavor and color is understood. We have assumed that the pion condensate aligns along the $\tau_{1}$ direction in flavor space. This choice is not restrictive. As a matter of fact we should allow for independent condensation both in $\pi^{+}$and in $\pi^{-}$channels [14]

$$
\pi^{+} \equiv\left\langle\bar{\psi} i \gamma_{5} \tau_{+} \psi\right\rangle=\frac{\pi}{\sqrt{2}} e^{i \theta}, \quad \pi^{-} \equiv\left\langle\bar{\psi} i \gamma_{5} \tau_{-} \psi\right\rangle=\frac{\pi}{\sqrt{2}} e^{-i \theta},
$$

with $\tau_{ \pm}=\left(\tau_{1} \pm \tau_{2}\right) / \sqrt{2}$; but the thermodynamical potential potential is not dependent on the phase $\theta$, therefore we can assume $\theta=0$ which leaves us with $\pi^{+}=\pi^{-}=\pi / \sqrt{2}$ and introduce only one condensate, specified in Eq. (9). In the mean field approximation the PNJL lagrangian reads

$$
\mathcal{L}=\bar{e}\left(i \gamma_{\mu} \partial^{\mu}+\mu_{e} \gamma_{0}\right) e+\bar{\psi}\left(i \gamma_{\mu} D^{\mu}+\hat{\mu} \gamma_{0}-M-N i \gamma_{5} \tau_{1}\right) \psi-G\left[\sigma^{2}+\pi^{2}\right]-\mathcal{U}[\Phi, \bar{\Phi}, T]
$$

where the in-medium quark mass $M$ and the pion field $N$ are related to $\sigma$ and $\pi$ by means of the relations

$$
\begin{aligned}
& M=m-2 G \sigma \\
& N=-2 G \pi .
\end{aligned}
$$

The thermodynamical potential $\Omega$ can be obtained by integration over the fermion fields in the partition function of the model, see for example Ref. [19],

$$
\Omega=-\left(\frac{\mu_{e}^{4}}{12 \pi^{2}}+\frac{\mu_{e}^{2} T^{2}}{6}+\frac{7 \pi^{2} T^{4}}{180}\right)+\mathcal{U}[\Phi, \bar{\Phi}, T]+G\left[\sigma^{2}+\pi^{2}\right]-T \sum_{n} \int_{0}^{\Lambda} \frac{d^{3} \boldsymbol{p}}{(2 \pi)^{3}} \operatorname{Tr} \log \frac{S^{-1}\left(i \omega_{n}, \boldsymbol{p}\right)}{T}
$$

where the sum is over fermion Matsubara frequencies $\omega_{n}=\pi T(2 n+1)$, and the trace is over Dirac, flavor and color indices. $\Lambda$ is an ultraviolet cutoff to ensure convergence of the momentum integral. The inverse quark propagator is defined as

$$
S^{-1}\left(i \omega_{n}, \boldsymbol{p}\right)=\left(\begin{array}{cc}
\left(i \omega_{n}+\mu-\frac{2}{3} \mu_{e}+i A_{4}\right) \gamma_{0}-\gamma \cdot \boldsymbol{p}-M & -i \gamma_{5} N \\
-i \gamma_{5} N & \left(i \omega_{n}+\mu+\frac{1}{3} \mu_{e}+i A_{4}\right) \gamma_{0}-\gamma \cdot \boldsymbol{p}-M
\end{array}\right) \otimes \mathbf{1}_{c}
$$

The ground state of the model is defined by the values of $\sigma, \pi, \Phi, \bar{\Phi}$ that minimize $\Omega$ and that have a vanishing total charge; the latter condition is equivalent to the requirement

$$
\frac{\partial \Omega}{\partial \mu_{e}}=0
$$

The parameters $m, G$ and $\Lambda$ are given by [7]

$$
m=5.5 \mathrm{MeV}, \quad G=5.04 \mathrm{GeV}^{-2}, \quad \Lambda=650.9 \mathrm{MeV},
$$

which fix, at zero temperature and zero chemical potential, the pion mass $m_{\pi}=139.3 \mathrm{MeV}$, the pion decay constant $f_{\pi}=92.3 \mathrm{MeV}$ and the chiral condensate $\langle\bar{u} u\rangle=-(251 \mathrm{MeV})^{3}$. 


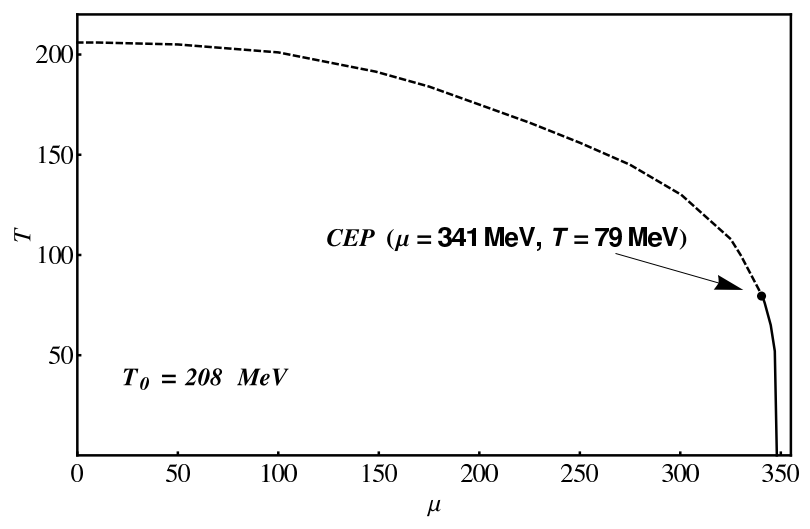

FIG. 1: Phase diagram of the electrically neutral two flavor PNJL model. Dashed line corresponds to the chiral crossover; solid line describes the first order chiral transition. At $\mu=0$ the critical point is located at $T=206 \mathrm{MeV}$; at $T=0$ the chiral transition is found at $\mu=348 \mathrm{MeV}$. The black dot denotes the critical end point (CEP), located at $\left(\mu_{E}, T_{E}\right)=(342,79) \mathrm{MeV}$.

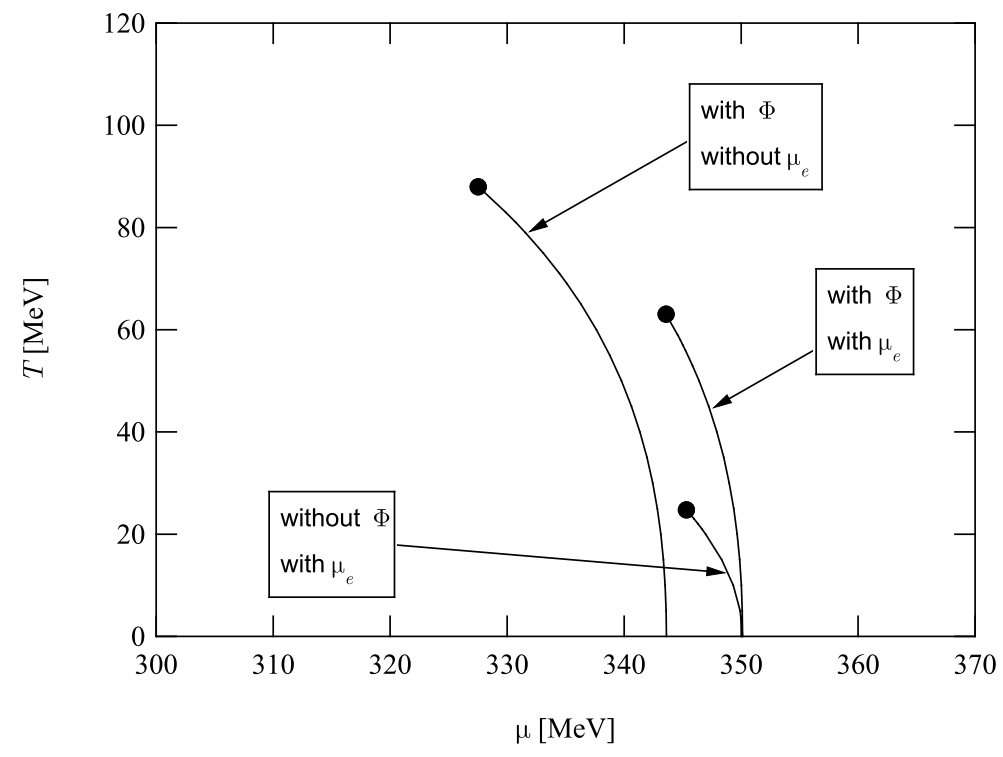

FIG. 2: Comparison of the first order transitions of the PNJL model with neutrality (called Model I in the text), of the PNJL model with $\mu_{e}=0$ (model II) and of the NJL model with electrical neutrality (model III).

\section{PHASE DIAGRAM OF THE MODEL}

In Fig. 1 we plot the phase diagram of the electrically neutral two flavor PNJL model. Dashed line corresponds to the chiral crossover; solid line describes the first order chiral transition. At each value of $\mu$ the crossover is identified with the inflection point of the chiral condensate. Analogously the first order transition is defined by the discontinuity of $\sigma$. In the region below the lines the chiral symmetry is broken, above the lines it is restored. At $\mu=0$ the chiral symmetry is restored at $T=206 \mathrm{MeV}$. For comparison, the inflection point of the Polyakov loop (which is commonly associated to the deconfinement crossover) at $\mu=0$ is located at $T=180 \mathrm{MeV}$. Moreover, at $T=0$ the chiral phase transition is of first order and is found at $\mu=348 \mathrm{MeV}$. The black dot denotes the critical end point (CEP), located at $\left(\mu_{E}, T_{E}\right)=(342,79) \mathrm{MeV}$. As explained later, we find a vanishing pion condensate once electrical neutrality is required.

It is instructive to compare the PNJL results discussed above with the electrically neutral NJL model, as well as the PNJL model with $\mu_{e}=0$, in order to emphasize the role of electrical neutrality and of the Polyakov loop. To this end in Fig. 2 we compare the first order transitions of the PNJL model with neutrality (model I), of the PNJL model with $\mu_{e}=0$ (model II) and of the NJL model with electrical neutrality (model III). Comparison of models I and II shows 

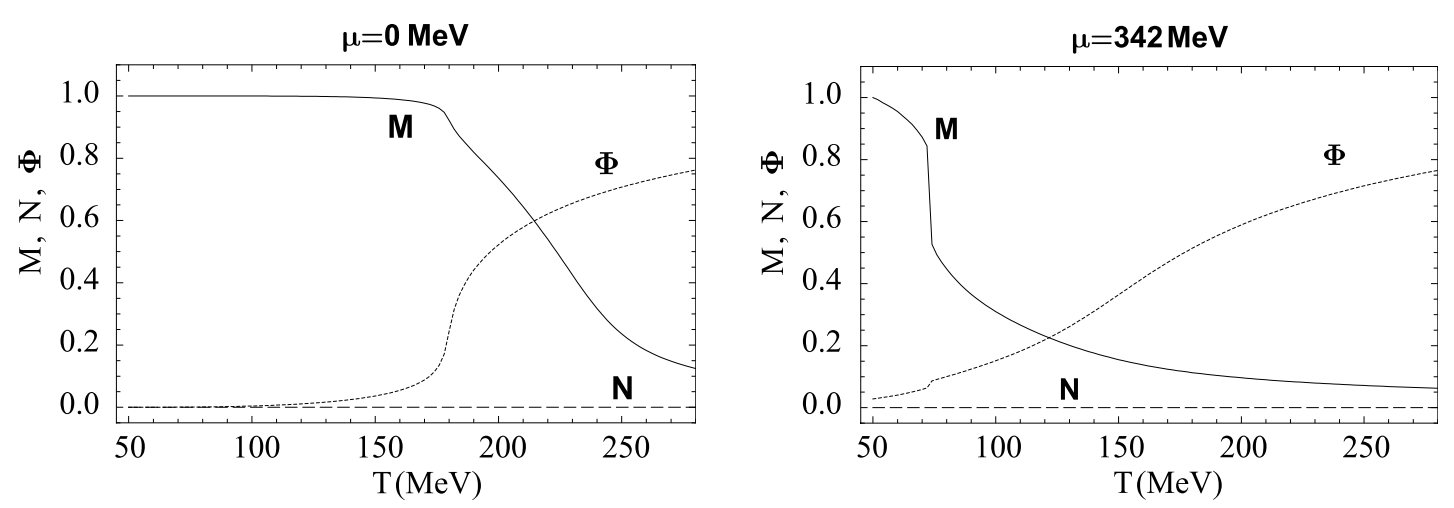

FIG. 3: Quark constituent mass $M$ (in units of $M_{0}$ ), pion condensate $N$ (in units of $M_{0}$ ) and $\Phi$ as a function of temperature (in $\mathrm{MeV}$ ) for different values of $\mu . M_{0}=325 \mathrm{MeV}$ is the constituent quark mass evaluated at $\mu=0, T=0$ and $\mu_{e}=0$.

that adding the electron chemical potential and requiring electrical neutrality enlarges of some $\mathrm{MeV}$ the $\mu$ window of the chirally broken phase. Moreover, comparison of models I and III shows that adding the self-consistently evaluated Polyakov loop to the model renders the broken phase more robust, as the critical temperatures are increased.

In Fig. 3 we plot the quark constituent mass $M$ (in units of $M_{0}$ ), pion condensate $N$ (in units of $M_{0}$ ) and $\Phi$ as a function of temperature (in $\mathrm{MeV}$ ) for two values of $\mu$. We have defined $M_{0}=325 \mathrm{MeV}$ as the constituent quark mass evaluated at $\mu=0, T=0$ and $\mu_{e}=0$. The electron chemical potential evaluated at some values of $\mu$ is shown in Fig. 4. We observe that when electrical neutrality is required, pions do not condense in the ground state of the model. As a matter of fact $N=0$ in Fig. 3] we have verified this result for several values of $\mu$. Stated in other words the isospin chemical potential $\mu_{I}=-\mu_{e} / 2$ in the neutral phase is always smaller than the value required for pion condensation to occur.

We have verified the robustness of our calculations in several ways. First, we have treated $\mu_{e}$ as a free parameter, reproducing the results of Ref. [14] finding a pion condensate for high values of $\mu_{e}$. Second, we have compared our results with those Ref. [18]. The authors of Ref. [18] study the neutral ground state in the NJL model at low temperature. They use a set of parameters in which the values of $G$ and $\Lambda$ are similar to ours, but they consider only the chiral limit $m_{u}=m_{d}=0$. In this limit they find a narrow window in $\mu$ at zero temperature in which the pion condensate can exist. We have explicitly checked that putting by hand $m_{u}=m_{d}=0$ in our calculations we reproduce the aforementioned window. This is a further check since at low temperature the PNJL reduces to the NJL model. Moreover we have verified that the pion condensate is washed out if the current quark mass is increased from zero to the physical value $m_{u}=m_{d}=5.5 \mathrm{MeV}$. This is explicitly shown in Fig 5 where we plot the pion condensate $N$ and the constituent quark mass $M$ in units of $M_{0}$, the constituent quark mass at $T=\mu=0$, as a function of the bare quark mass $m_{0}$ at the representative value of $\mu=330 \mathrm{MeV}$ (we have checked that the same result hold for other values of $\mu$ ) and $T=0$. Therefore the current quark mass works as an external field that drives the alignment of the vacuum along the chiral condensate direction. We stress that Fig. 5 has to be taken only as a trick that allows a clear comparison between our results and those of Ref. [18]. A true plot of $M$ and $N$ as a function of the current quark mass requires the self consistent evaluation of $G$ and $\Lambda$, which is beyond the scope of the present paper since we study only the phase diagram and the pion modes at the physical point. Nevertheless the problem of the modification of the ground state by variations of the current quark mass is interesting and deserves further study, therefore we leave this to a future project.

\section{PSEUDO-GOLDSTONE MODES}

In this Section we compute the masses of the pseudo-Goldstone modes as well as of the $\sigma$ mode in the two flavor and electrically neutral PNJL model. As discussed in the previous Section for each value of $\mu$ and $T$, after requiring electrical neutrality, the difference of chemical potentials between up and down quarks is lower than the critical value required for the realization of the pion condensation. In this Section we therefore put by hand $N=0$ in the quark propagator, Eq. (15).

The pion mass is defined as the solution of the well-known Bethe-Salpeter equation for the bound states. Its derivation in the context of the NJL model is in Ref. [2]. The same equation is valid in the PNJL model, the difference between PNJL and NJL being only the different quark propagator to be used in the calculation of the pion 


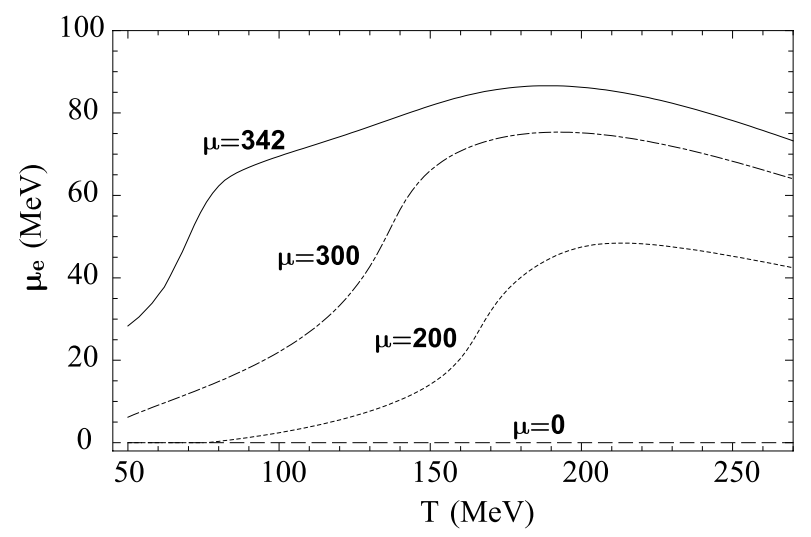

FIG. 4: Electron chemical potential as a function of the temperature, for different values of the quark chemical potential.

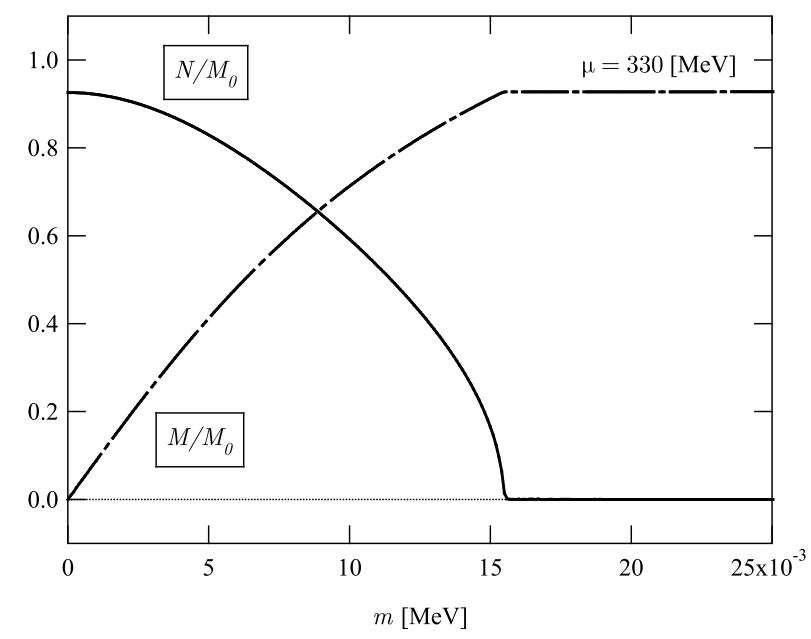

FIG. 5: Pion condensate $N$ and the constituent quark mass $M$ in units of $M_{0}$, the constituent quark mass at $T=\mu=0$, as a function of the bare quark mass $m_{0}$ at the representative value of $\mu=330 \mathrm{MeV}$ and $T=0$.

polarization tensor. The equation reads

$$
1-2 G \Pi_{A}\left(m_{A}^{2}\right)=0, \quad A=1,2,3 .
$$

In the above equation $\Pi_{A}$ is the polarization tensor of the pion $A$, specified later, where $A=1,2,3$ correspond respectively to $\pi^{+}, \pi^{-}$and $\pi^{0} ; m_{A}$ denotes the pion mass, and $G$ is the coupling constant introduced in Eq. (77). The solutions of Eq. (18) correspond to the poles of the pion propagator, the latter evaluated in the random phase approximation [2].

We now specify the pion polarization tensor. We introduce the polarization matrix

$$
\Pi_{i j}\left(m^{2}\right)=-i \int \frac{d^{4} p}{(2 \pi)^{4}} \operatorname{Tr}\left[i \gamma_{5} \tilde{\tau}_{i} i S\left(p+\frac{k}{2}\right) i \gamma_{5} \tilde{\tau}_{j} i S\left(p-\frac{k}{2}\right)\right]
$$

here $i, j=1,2,3, k_{\mu}=(m, \mathbf{0})$, and the trace is over color, flavor and Dirac indices; the matrices $\tilde{\tau}_{i}$ are operators in the flavor space, defined by

$$
\tilde{\tau}_{1,2}=\frac{1}{\sqrt{2}}\left(\tau_{1} \pm i \tau_{2}\right), \quad \tilde{\tau}_{3}=\tau_{3},
$$

where $\tilde{\tau}_{i}, i=1,2,3$ are the Pauli matrices; $S$ is the fermion propagator, whose inverse is defined in Eq. (15) with $N=0$, and masses and chemical potentials evaluated self consistently in the electrically neutral phase. In terms of 

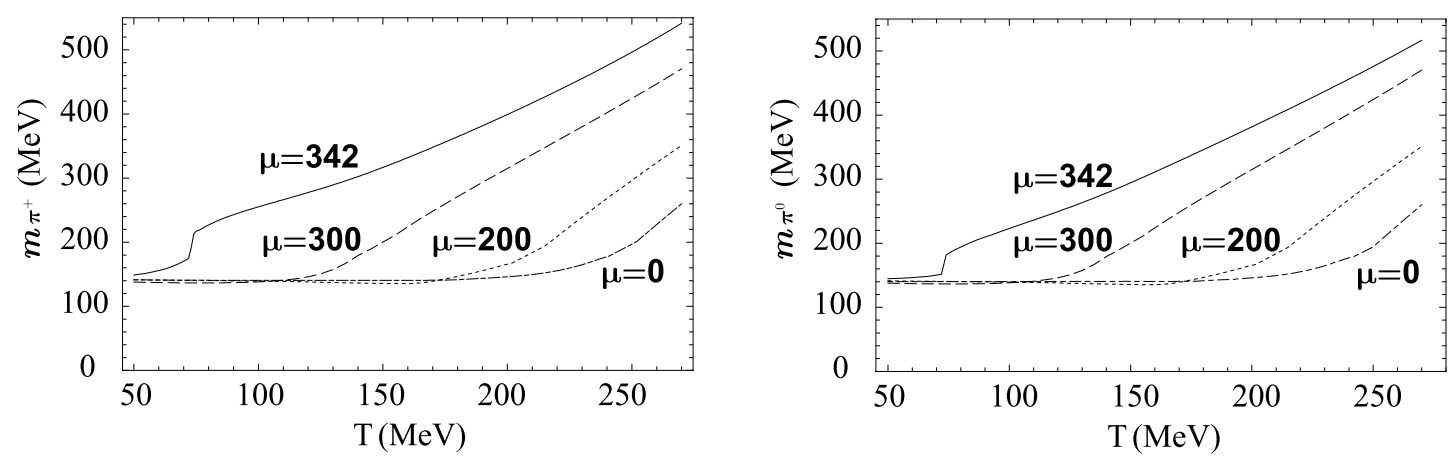

FIG. 6: Masses of the charged pions (left panel) and of the neutral pion (right panel) in the electrically neutral phase, as a function of the temperature, for different values of the quark chemical potential.

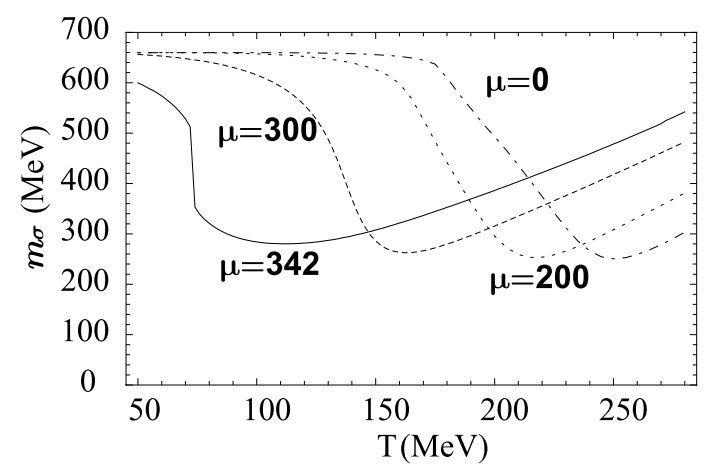

FIG. 7: Mass of the $\sigma$ mode in the electrically neutral phase, as a function of the temperature, for different values of the quark chemical potential.

the matrix $\Pi_{i j}$ the pion polarization tensors appearing in Eq. (19) are defined as

$$
\Pi_{1}=\Pi_{12}, \quad \Pi_{2}=\Pi_{21}, \quad \Pi_{3}=\Pi_{33} .
$$

Analogously the equation for the $\sigma$ mass reads

$$
1-2 G \Pi_{\sigma}\left(m_{\sigma}^{2}\right)=0
$$

with the polarization tensor defined as

$$
\Pi_{\sigma}\left(m^{2}\right)=-i \int \frac{d^{4} p}{(2 \pi)^{4}} \operatorname{Tr}\left[i S\left(p+\frac{k}{2}\right) i S\left(p-\frac{k}{2}\right)\right] .
$$

Once the traces in Eqs. (18) and (22) are evaluated, we are left with the loop 4-momentum integral. We replace the integral over energy by a summation over fermion Matsubara frequencies; moreover, the integral over directions of momentum is trivial. Therefore we are left with the integration over the modulus of $\boldsymbol{p}$ only, which is evaluated numerically.

In Fig. 6] we plot the masses of the charged pions (left panel) and of the neutral pion (right panel) in the electrically neutral phase, as a function of the temperature, for different values of the quark chemical potential. The behavior of the pseudoscalar modes as the temperature is increased is qualitatively the same observed in the NJL model [2]. At each value of $\mu$, the charged and neutral pion masses are of the order of the zero temperature value $\approx 140 \mathrm{MeV}$ below the chiral crossover (or the chiral first order transition); as the chiral transition (either first order or crossover) occurs, the pion masses rapidly increase signaling the disappearing of the pseudoscalar modes from the low energy spectrum of the model.

In Fig. 7 we plot the mass of the $\sigma$ mode in the electrically neutral phase, as a function of the temperature, for different values of the quark chemical potential. As in the case of the pions, the behavior of $m_{\sigma}$ is analogous to that observed in the NJL model [2]. 

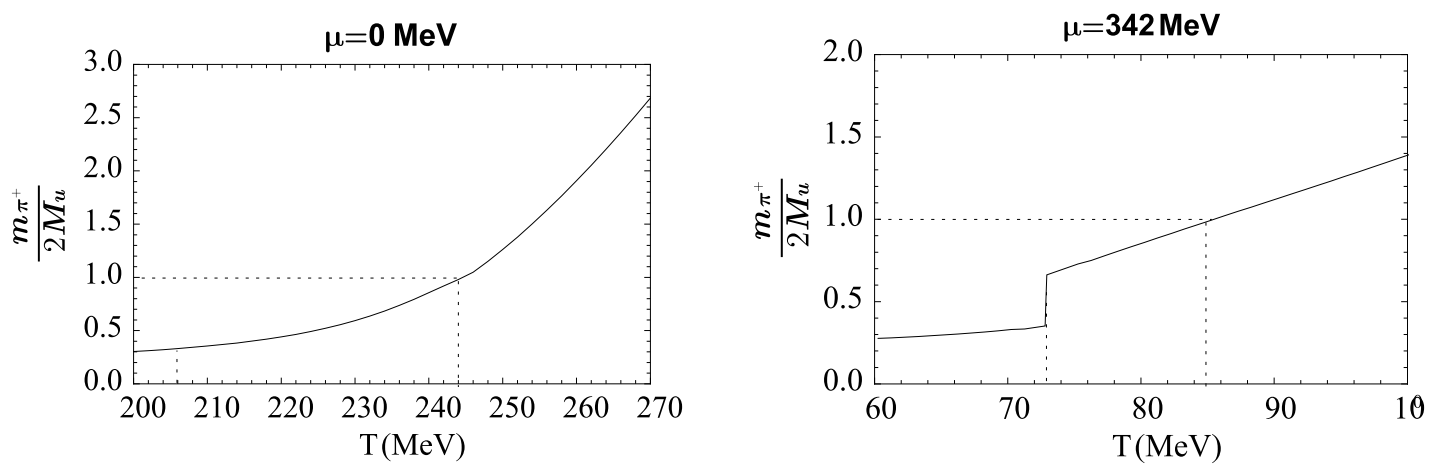

FIG. 8: The ratio $m_{\pi} / 2 M$, with $M$ the constituent quark mass, as a function of temperature, for two representative values of the quark chemical potential. The vertical bold dashed line denotes the chiral transition temperature.

\begin{tabular}{|c|c|c|c|c|c|c|}
\hline & $T_{\chi}\left(\mu_{e}=0\right)$ & $T_{M}\left(\mu_{e}=0\right)$ & $T_{M} / T_{\chi}\left(\mu_{e}=0\right)$ & $T_{\chi}$ & $T_{M}$ & $T_{M} / T_{\chi}$ \\
\hline$\mu=100$ & 218 & 236 & 1.08 & 198 & 234 & 1.18 \\
\hline$\mu=200$ & 178 & 207 & 1.16 & 175 & 211 & 1.20 \\
\hline$\mu=300$ & 131 & 145 & 1.10 & 131 & 154 & 1.17 \\
\hline$\mu=342$ & 44 & 48 & 1.09 & 73 & 85 & 1.16 \\
\hline
\end{tabular}

TABLE I: Comparison of the pion melting temperatures $T_{M}$ and the chiral transition temperatures $T_{\chi}$ with electrical neutrality and with $\mu_{e}=0$. Quark chemical potentials and temperatures are measured in $\mathrm{MeV}$.

It is interesting to evaluate the ratio $m_{\pi} / 2 M$, with $M$ the constituent quark mass, as a function of temperature. If it is larger than one, than a bound state with the quantum numbers of the pion is less stable than a state made of a free quark and a free antiquark, and thus the pion melts to its constituent quarks. In Fig. 8 we show the results of the computation of $m_{\pi} / 2 M$ at different values of $\mu$ in the electrically neutral phase, for values of temperatures close either to the chiral crossover or to the first order chiral transition. We notice that for any value of the quark chemical potential there exists a window in temperature, above the chiral restoration temperature, in which the pions are lighter than a quark and an antiquark. The window shrinks as $\mu$ is increased. These results show that even above the critical temperature a bound state with the pions quantum numbers can be formed.

We observe that the window in which the bound state is stable above the chiral phase transition (as well as the chiral crossover) is not a peculiarity of the PNJL model. As a matter of fact the same window was noticed in the NJL model at $\mu=0$ in Ref. [20]. We have verified that it exists in the neutral NJL model as well. For example, at $\mu=0$ we find that the bound states exist in the window $(195,212) \mathrm{MeV}$; this result has to be compared with the PNJL one, namely $(205,245) \mathrm{MeV}$, showing that the PNJL window is almost 2.4 times larger than the NJL one. Analogously at $\mu=342 \mathrm{MeV}$ the NJL window is $(34,44) \mathrm{MeV}$, to be compared with the PNJL result $(73,85) \mathrm{MeV}$.

Similar results have been obtained in the framework of the PNJL model in Ref. [21]. The authors of Ref. 21] consider the two flavor PNJL model without the constraint of electrical neutrality. Moreover they use a polynomial form of the Polyakov loop effective potential $\mathcal{U}$ in Eq. (77) instead of the logarithmic one, see Eq. (3). Therefore a quantitative comparison with their results is not feasible. Instead we have computed the ratio $T_{M} / T_{\chi}$ in the case $\mu_{e}=0$ in the PNJL model with the logarithmic form of the effective potential, for several values of $\mu$, in order to quantify the role of the electrical neutrality on the ratio $T_{M} / T_{\chi}$. Our results are summarized in Table I.

It is interesting to superimpose the bound state existence region to the phase diagram in Fig. 11 This is done in Fig. 9 where we show the region in the $\mu-T$ plane where the bound state can be formed. In the white region below the gray domain the chiral symmetry is broken and pions live as pseudo-Goldstone modes. In the gray region chiral symmetry is restored but $m_{\pi} / 2 M_{q}<1$, thus bound states can be formed. Finally, in the white region above the gray domain chiral symmetry is restored and free quark states are more stable than bound states (this region is characterized by $\left.m_{\pi} / 2 M_{q}>1\right)$.

The existence of bound states above the chiral transition temperature is interesting because it implies that quark matter above the chiral transition is strongly coupled. It has been suggested [22, 23] that the formation of bound states just above the chiral transition can explain the experimental results obtained by non-central heavy ion collisions at RHIC facility [24, 25, 26, 27]; in particular it provides a simple explanation to the low viscosity-to-entropy ratio 
observed in elliptic flow simulations. The next step would be the computation of the transport coefficients of quark matter just above the chiral transition temperature. We leave this to a future project.

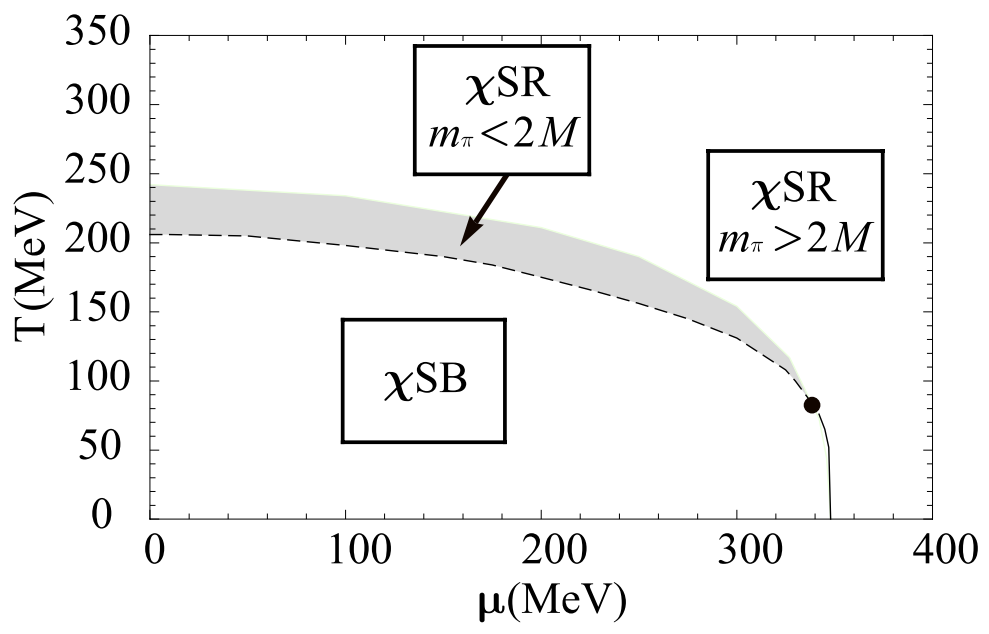

FIG. 9: Region of existence of bound states in the $\mu-T$ plane. $\chi S B$ denotes the region where chiral symmetry is broken; $\chi S R$ denotes the region where chiral symmetry is restored. $M$ is the constituent quark mass. The dashed and the solid lines correspond respectively to the chiral crossover and to the chiral first order transition, the dot denotes the CEP. In the gray region chiral symmetry is restored but a bound state with the quantum numbers of the pions can still be formed. See the text for more details.

\section{CONCLUSIONS}

In this paper we have studied the two flavor PNJL model in presence of a charge chemical potential, requiring electrical neutrality in the mean field approximation. We have assumed that in the ground state both the chiral and the pion condensates develop, and we have evaluated their values, as well as the value of the Polyakov loop, for each value of the temperature and of the quark chemical potential by minimizing the thermodynamic potential under the condition that the total electric charge of the system vanishes.

We have drawn the phase diagram of the model, see Fig. 1. One of our main results is that we do not observe pion condensation in the mean field approximation. We relate this to the non vanishing current quark mass, as shown in Fig. 5. This part of our study is completed by the results for the constituent quark masses, the Polyakov loop and the charge chemical potential, see Figs. 3 and 4 .

We have computed the masses of the pseudo-Goldstone modes and of the $\sigma$-mode. The behavior of the masses as a function of the temperature for different values of the quark chemical potential are shown in Figs. [and 7 Furthermore we have investigated on the possibility of existence of bound states with the quantum numbers of the pions above the chiral critical temperature. To this end we have compared the computed pion mass with twice the constituent quark mass. The result is shown in Fig. 8. We have found that for any value of the quark chemical potential there exists a window in temperature, above the chiral restoration temperature, in which the pions are lighter than a state formed by a free quark and a free antiquark. The aforementioned window shrinks as $\mu$ is increased. Our results show that even above the critical temperature a bound state with the pions quantum numbers can be formed. This is summarized in Fig. 9

In this work we have not studied independently the role of the chemical potential on the suppression of the pion condensate. This can be done by leaving the isospin chemical potential as a fixed and free parameter, and varying only the mean quark chemical potential. A further natural extension of our work is the study of the $2+1$ flavor PNJL model with meson condensation, with and without neutrality conditions implemented. Moreover, it would be interesting to include a color superconductive state in the neutral model [28] (studies of the superconductive state without neutrality conditions can be found in Refs. [7, 15]). Even more one should compute the transport coefficients 
of quark matter just above the chiral transition temperature. We leave these projects to future works.

[1] Y. Nambu and G. Jona-Lasinio, Phys. Rev. 122, 345 (1961); Phys. Rev. 124, 246 (1961).

[2] S. P. Klevansky, Rev. Mod. Phys. 64, 649 (1992); T. Hatsuda and T. Kunihiro, Phys. Rept. 247, 221 (1994) arXiv:hep-ph/9401310; M. Buballa, Phys. Rept. 407, 205 (2005) arXiv:hep-ph/0402234.

[3] A. M. Polyakov, Phys. Lett. B 72, 477 (1978); L. Susskind, Phys. Rev. D 20, 2610 (1979); B. Svetitsky and L. G. Yaffe, Nucl. Phys. B 210, 423 (1982); B. Svetitsky, Phys. Rept. 132, 1 (1986).

[4] P. N. Meisinger and M. C. Ogilvie, Phys. Lett. B 379, 163 (1996) arXiv:hep-lat/9512011].

[5] K. Fukushima, Phys. Lett. B 591, 277 (2004) arXiv:hep-ph/0310121.

[6] C. Ratti, M. A. Thaler and W. Weise, Phys. Rev. D 73, 014019 (2006) arXiv:hep-ph/0506234.

[7] S. Roessner, C. Ratti and W. Weise, Phys. Rev. D 75, 034007 (2007) arXiv:hep-ph/0609281.

[8] S. K. Ghosh, T. K. Mukherjee, M. G. Mustafa and R. Ray, arXiv:0710.2790 [hep-ph]; S. K. Ghosh, T. K. Mukherjee, M. G. Mustafa and R. Ray, Phys. Rev. D 73, 114007 (2006) arXiv:hep-ph/0603050.

[9] K. Kashiwa, H. Kouno, M. Matsuzaki and M. Yahiro, arXiv:0710.2180 [hep-ph].

[10] B. J. Schaefer, J. M. Pawlowski and J. Wambach, Phys. Rev. D 76, 074023 (2007) arXiv:0704.3234 [hep-ph]].

[11] C. Ratti, S. Roessner and W. Weise, Phys. Lett. B 649, 57 (2007) arXiv:hep-ph/0701091.

[12] C. Sasaki, B. Friman and K. Redlich, Phys. Rev. D 75, 074013 (2007) arXiv:hep-ph/0611147; C. Sasaki, B. Friman and K. Redlich, Phys. Rev. D 75, 054026 (2007) arXiv:hep-ph/0611143.

[13] E. Megias, E. Ruiz Arriola and L. L. Salcedo, Phys. Rev. D 74, 114014 (2006) arXiv:hep-ph/0607338; E. Megias, E. Ruiz Arriola and L. L. Salcedo, Phys. Rev. D 74, 065005 (2006) arXiv:hep-ph/0412308.

[14] Z. Zhang and Y. X. Liu, Phys. Rev. C 75, 064910 (2007) arXiv:hep-ph/0610221.

[15] M. Ciminale, G. Nardulli, M. Ruggieri and R. Gatto, Phys. Lett. B 657, 64 (2007) arXiv:0706.4215 [hep-ph]].

[16] W. j. Fu, Z. Zhang and Y. x. Liu, Phys. Rev. D 77, 014006 (2008) arXiv:0711.0154 [hep-ph]].

[17] M. Ciminale, R. Gatto, N. D. Ippolito, G. Nardulli and M. Ruggieri, arXiv:0711.3397] [hep-ph].

[18] D. Ebert and K. G. Klimenko, Eur. Phys. J. C 46, 771 (2006) arXiv:hep-ph/0510222.

[19] D. Ebert, K. G. Klimenko and H. Toki, Phys. Rev. D 64, 014038 (2001) arXiv:hep-ph/0011273; D. Ebert, V. V. Khudyakov, V. C. Zhukovsky and K. G. Klimenko, Phys. Rev. D 65, 054024 (2002) arXiv:hep-ph/0106110.

[20] T. Hatsuda and T. Kunihiro, Phys. Rev. Lett. 55 (1985) 158.

[21] H. Hansen, W. M. Alberico, A. Beraudo, A. Molinari, M. Nardi and C. Ratti, Phys. Rev. D 75, 065004 (2007) arXiv:hep-ph/0609116.

[22] E. V. Shuryak and I. Zahed, Phys. Rev. C 70, 021901 (2004) arXiv:hep-ph/0307267.

[23] G. E. Brown, C. H. Lee, M. Rho and E. Shuryak, Nucl. Phys. A 740, 171 (2004) arXiv:hep-ph/0312175].

[24] P. F. Kolb and U. W. Heinz, arXiv:nucl-th/0305084.

[25] D. Molnar and M. Gyulassy, Nucl. Phys. A 697, 495 (2002) [Erratum-ibid. A 703, 893 (2002)] arXiv:nucl-th/0104073.

[26] D. Teaney, Phys. Rev. C 68, 034913 (2003) arXiv:nucl-th/0301099.

[27] A. Nakamura and S. Sakai, Phys. Rev. Lett. 94, 072305 (2005) arXiv:hep-lat/0406009; A. Peshier and W. Cassing, Phys. Rev. Lett. 94, 172301 (2005) arXiv:hep-ph/0502138.

[28] R. Rapp, T. Schafer, E. V. Shuryak and M. Velkovsky, Phys. Rev. Lett. 81, 53 (1998) arXiv:hep-ph/9711396; M. G. Alford, K. Rajagopal and F. Wilczek, Phys. Lett. B 422, 247 (1998) arXiv:hep-ph/9711395; M. G. Alford, K. Rajagopal and F. Wilczek, Nucl. Phys. B 537, 443 (1999) arXiv:hep-ph/9804403. 